\title{
Fatigue Analysis of Automobile Control Arm Based on Ncode
}

\author{
Ren Huanmei ${ }^{1}$ and Chen Lihui ${ }^{2}$ \\ ${ }_{1}^{1}$ No. 1111, Hucheng Ring Road, Pudong New District Shanghai City, PRC \\ ${ }^{2}$ No. 145, Tiantai Road, Hantan District, Hanzhong, Shaanxi, PRC
}

\begin{abstract}
In order to improve the vehicle chassis structure durability, the fatigue analysis and optimization design of the low control arm (LCA) was taken. A finite element model was established. By using this model, the stress distribution and lowest point of lifetime of the control arm under fatigue load was calculated. Based on the results of analysis, the optimization scheme according to the structure characteristics of components was presented, and a solution to improve the control arm fatigue life was given out. The research provided reference for engineering application of calculation and optimization of chassis components fatigue life.
\end{abstract}

\section{Introduction}

Suspension system plays an important role in the chassis structure, it transfers force and torque between the wheels and the body, a guiding and controlling the relative movement of the wheel and body. It mitigates body shock transferred from the road and attenuates vibration of system and so on. Therefore, the suspension design is very important in automobile chassis design and the development of automobiles. McPherson suspension system has the advantages of compact structure, little change of wheel alignment parameters with beating of wheels, stability, and due to the cancellation of the upper arm, the arrangement of engine and steering system is convenient [1]. It is a durable independent suspension of with strong ability to adapt to the road. It has been used most widely as the independent suspension in the car.

In early stage of product development, it is important to focus on investigation of the structure strength and fatigue life of the key pieces. Suspension is an important force transferring system, its strength and fatigue life is required to be very strict. For McPherson independent suspension, the lower arm is the affordability of the subject [2]. Therefore the strength and service life of lower arm play an important role in suspension work [3]. Reliable prediction of fatigue life, analysis of influence factors of fatigue life, are of great significance to guide the design.

\section{Fatigue load}

For calculation, there are two categories of fatigue load named classic fatigue load and fatigue load spectrum [4]. Classic fatigue condition is defined as conditions in certain standards and specifications based on experience and accumulation, which is combinations of vertical beating, lateral impact, longitudinal impact, steering (left and right), braking and acceleration (left and right). Classic fatigue condition depends on long time accumulation and maturity standards. The standard load spectrum is measurements of the direct or indirect timevarying load at hard points of components.

The load spectrum are from the following: 1) Large vehicle factory's load spectrum accumulation of vehicles on the same platform. These measured spectrum, multiplied by a coefficient, can be used as a initial assessment of fatigue life in the early stage of the development of new models. 2) Directly measuring load spectrum of concerned parts. Due to measurement on a single component of single model, this method has a disadvantage of poor. 3) To measure spectrum of the wheel core with Six Axis Force method, then through load decomposition to obtain the load spectrum of concerned parts is obtained[7]. As this paper is for a new vehicle model development, the classic fatigue condition method was used to calculate fatigue life of the control arm.

\section{Control arm fatigue life prediction}

There are two categories of fatigue life calculation method commonly used. 1) Based on S-N curve, the fatigue life was calculated through application of linear fatigue cumulative damage rule. This method is also called nominal stress approach, or SN approach. 2) Get life of crack with stress-strain estimation, then added with crack propagation life from fracture mechanics to get total life $[5,6]$. This is called EN method. SN method is applied in high cycle fatigue, EN method is applied in low cycle fatigue. For automobile chassis component, most of the damage is low cycle fatigue failure, so we used EN method to calculate the fatigue life[7]. 


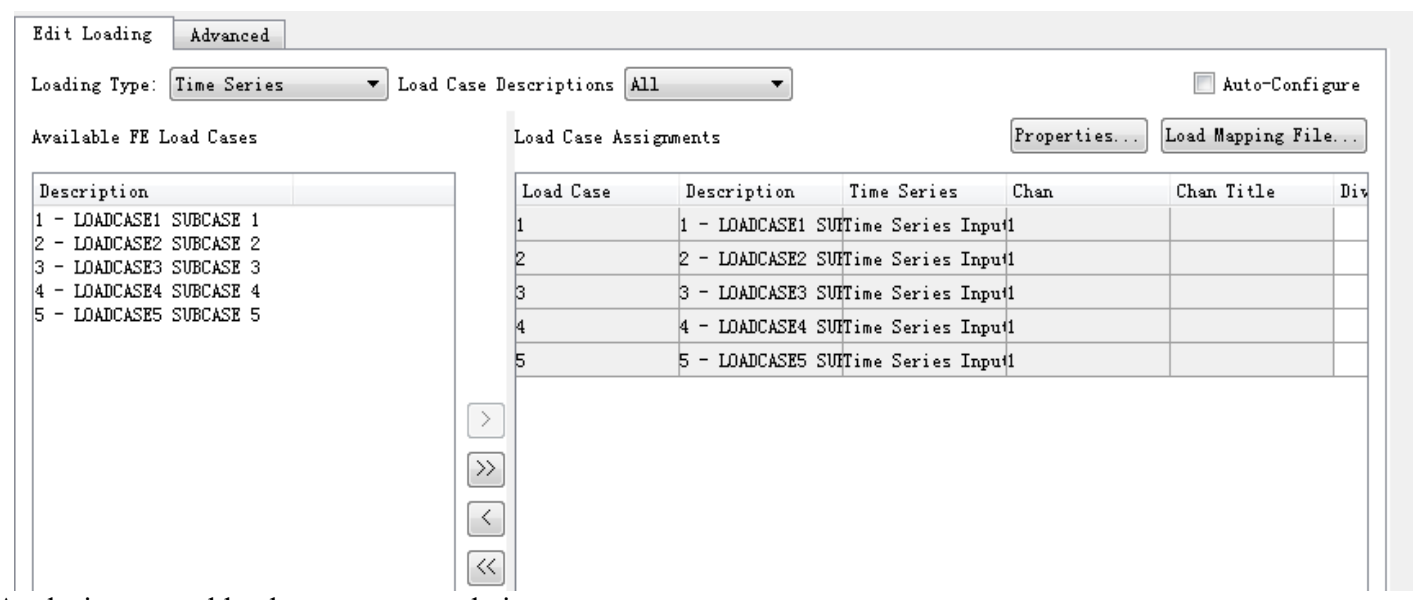

Figure 1. Analysis step and load spectrum correlation.

\subsection{Setting fatigue load}

The stress results were calculated for control arm under fatigue condition by using Natran linear solver. The results file was put into Ncode software as the input file of fatigue calculation. The half sine wave is set simulating bench test loading process. The waveform curve loading and the analysis step are correlated to simulating a drive cycle [8], [9]. The vertical beating, lateral impact, longitudinal impact, steering (left and right), brake and acceleration were respectively cyclic loaded once as a big cycle. The correlation results are shown in Figure 1.

\subsection{Material parameters}

Upon experience, SAPH440 was used for the control arm with reference the same vehicle classification. Material parameters of SAPH440 was input by using material editor in Ncode. The stress-strain curve was generated, which is shown in Figure 2. The result was used to solve the fatigue life of the control arm.

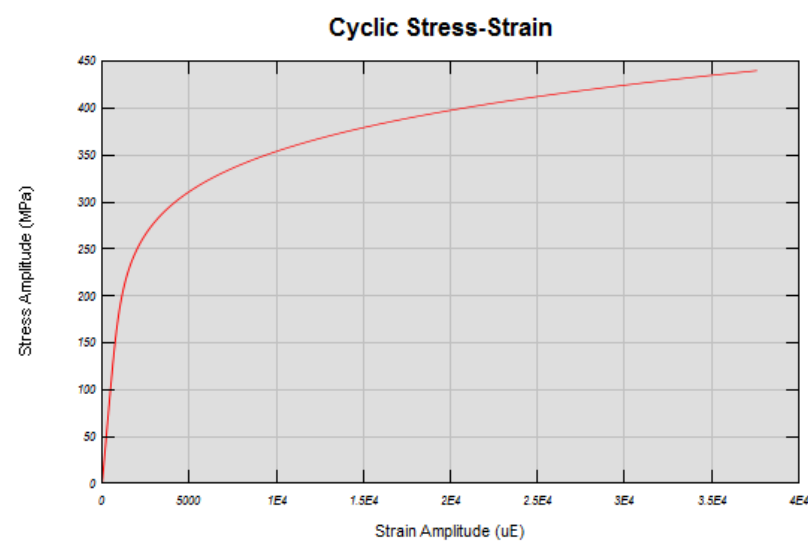

Figure 2. Stress-strain curves of SAPH440.

The output was set up to complete the fatigue process. The complete solving process was shown in Figure 3.

According to the calculation result, the control arm had a minimum life cycle of $1.5 \mathrm{E}+5$, which did not meet the life target of $1 \mathrm{E}+6$ cycle. The minimum life presents in the intensive board. Therefore, it is necessary to optimize the design of reinforcing plate in order to meet the minimum life target.

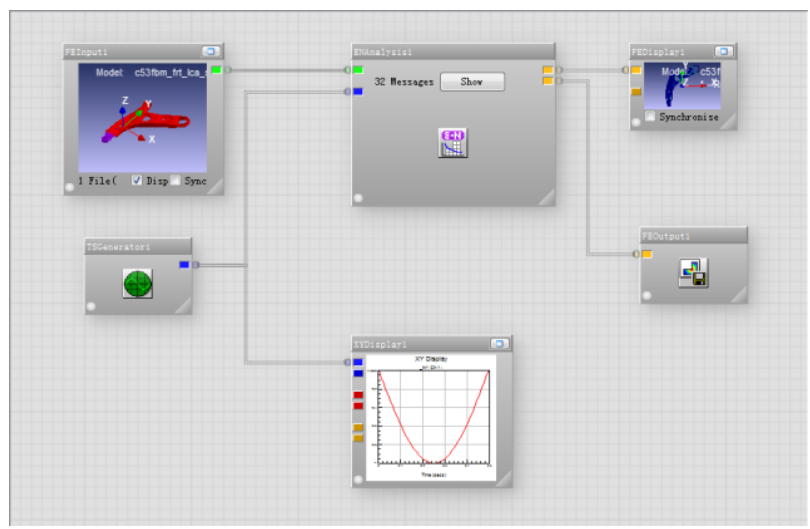

Figure 3. Flow chart of fatigue calculation in Ncode.

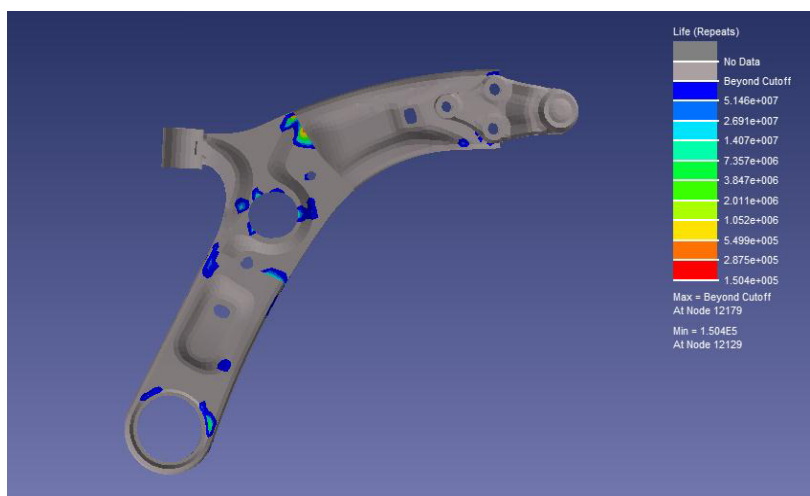

Figure 4. Calculated fatigue life of control arm.

As results of analysis, life of the control arm body should be more than $1 \mathrm{E}+7$ cycle, only local area of lower reinforcement did not meet the minimum life requirements. Lower life in this area was maily due to unreasonable structure. The bevel was too close to the weld bead, causing stress concentration in local area, which led to low fatigue life.

The original control arm body was retained, meanwhile the reinforcing plate was optimized [10]. The bevel of the reinforcing plate was shifted away from end of the weld bead. This migrated the stress concentration in the area. Resulted structure is shown in Figure 5. 


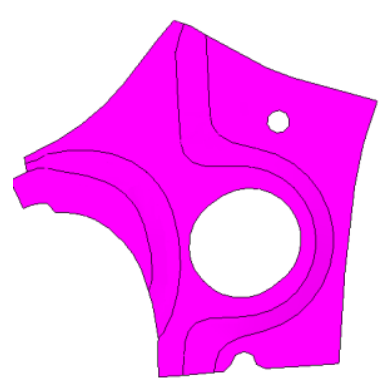

Before optimization

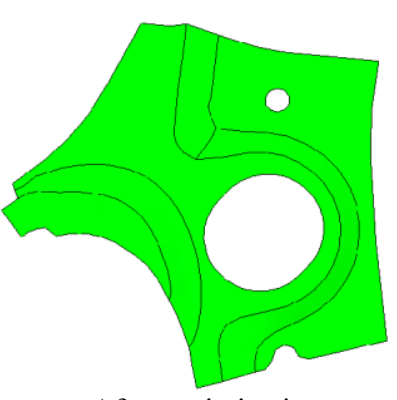

After optimization
Figure 5. Comparison of the reinforcing plate before and after optimization.

\subsection{Optimization and evaluation}

Optimization of the bevel position in reinforcing plate effectively migrated stress concentration with very little effect on other parts of the control arm. Fatigue life of this area was increased to $1.1 \mathrm{E}+6$ cycle. After the optimization, control arm had lowest life of $1.1 \mathrm{E}+6$ cycles, and fatigue life of the vast majority of the arm were higher than $7 \mathrm{E}+7$ cycle. After optimization, control arm fatigue life met the target. The lifetime results of optimization is shown in Figure 6.

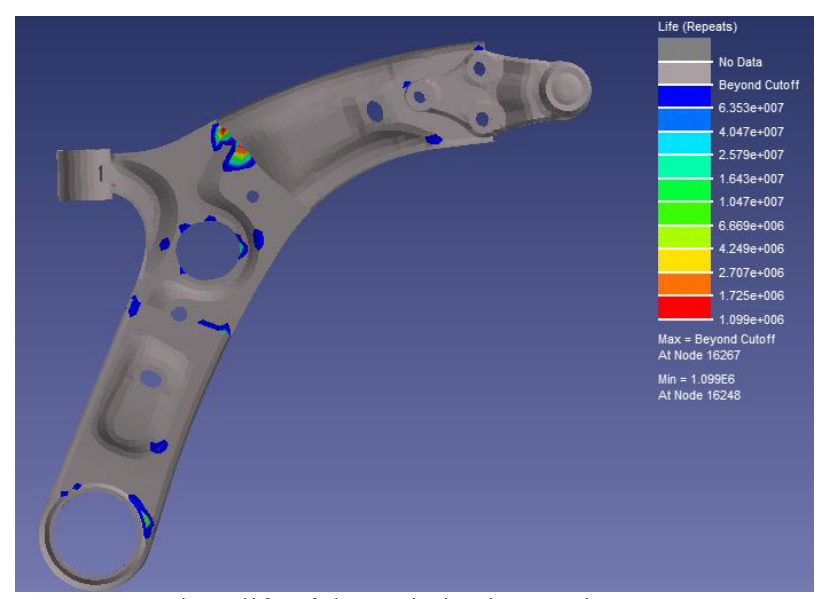

Figure 6. Fatigue life of the optimized control arm.

\section{Conclusions}

In this paper, a basic method of fatigue analysis and fatigue life prediction of control arm in early stage of vehicle development is presented, the minimum fatigue life was determined through calculation of control arm's fatigue life. The optimization scheme was presented on basis of this. With this scheme, making of control arm meeting the application requirements will be assured. The method allows fatigue life of control arm to be predicted and design to be optimized in early stage of the product development, the design of product also can be optimized upon it. This greatly reduced the product development cycle [11]. It is of significant importance to direct the engineering design.

\section{References}

1. Liu Yongchen, Wang Guolin, Sun Li. Fatigue damage analysis and life prediction for vehicle control arm. Journal of agricultural engineering. Vol. 35, 57-59 (2013)

2. Liu Yongchen, Wang Guolin, Sun Li. Reserch of the Vehicle Front Suspension Assembly Durability Simulation. The Fifth China CAE Annual Conference. 173-177 (2009)

3. Nagendra Iranna Jamadar and K K DhandeTransient. Analysis of Vehicle Passive Suspension System. IJMERR. Volume 3, No. 3, July, 451-464 (2014)

4. Huang Minfeng, Jiang Chun. Analyzing fatigue life of automobile components based on FEM. Mechanical Research \& Application. Vol. 21, 57-60 (2008)

5. Zhang Haibo, Zhang Ruijun. A Finite Element Analysis of Automobile Suspension Control Arm Base on ANSYS. Modular Machine Tool \& Automatic Manufacturing Technique. Vol. 45, 42-45 (2015)

6. Thanh-Tung, Tran, Hirosh. Advanced Passive Suspension with Inerter Devices and Optimization Design for Vehicle Oscillation. IJMERR. Volume 4, No. 4, October, 354-360 (2015)

7. HB Zhang, RJ Zhang, Y Chang. Finite Element Analysis of Automobile Suspension Control Arm. Applied Mechanics \& Materials. Vol. 752-753, 859863 (2015)

8. BC Song, HS Park, YM Kwon. YC Park: Structural Design of an Upper Control Arm, Considering Static Strength. Transactions of the Korean Society of Automotive Engineers. Vol. 17, 190-196 (2009)

9. B Ashtekar and A G Thakur. Simulink Model of Suspension System and its Validation on Suspension Test Rig. IJMERR. Volume 3, No. 3, July, 811-818 (2014)

10. Maotao Zhu, Jiahuan Guo, Tao Liu. Finite Element Analysis and Fatigue Analysis of Control Arm. The Series Lecture Notes in Electrical Engineering. Vol. 364, 457-468 (2015)

11. Raghav Goyal, Anitej Tripathy, Mandeep Singh Gujral. Producing Suspension. IJMERR. Volume 3, No. 2, April, 239-244 (2014) 